\title{
Bupivacaine inhibits the malignant biological behavior of oral squamous cell carcinoma cells by inhibiting the activation of ERK1/2 and STAT3
}

\author{
Zhongchao Wang $^{1,2 \#}$, Jie Zhang ${ }^{3 \#}$, Yongmei Wen ${ }^{1,4}$, Pin Wang ${ }^{1,4}$, Liyuan Fan ${ }^{1,4}$ \\ ${ }^{1}$ Oral \& Maxillofacial Reconstruction and Regeneration Laboratory, Southwest Medical University, Luzhou, China; ${ }^{2}$ Department of Periodontics \\ \& Oral Medicine, The Affiliated Hospital of Stomatology of Southwest Medical University, Luzhou, China; ${ }^{3}$ Medical Administration Department, \\ Hospital of Chengdu University of Traditional Chinese Medicine, Chengdu, China; ${ }^{4}$ Department of Prosthodontics, The Affiliated Hospital of \\ Stomatology of Southwest Medical University, Luzhou, China \\ Contributions: (I) Conception and design: Z Wang, J Zhang, L Fan; (II) administrative support: Y Wen; (III) provision of study materials or patients: Z \\ Wang, Y Wen, J Zhang, P Wang; (IV) collection and assembly of data: All authors; (V) data analysis and interpretation: Y Wen, J Zhang, P Wang, L \\ Fan; (VI) manuscript writing: All authors; (VII) final approval of manuscript: All authors. \\ "These authors contributed equally to this work. \\ Correspondence to: Liyuan Fan. No. 2 Jiang Yang Nan Lu, Luzhou, China. Email: fanliyuan_8356@163.com.
}

Background: Oral squamous cell carcinoma (OSCC) is an aggressive malignant tumor. Bupivacaine (Bupi), a local anesthetic drug, has been shown to display anti-tumor activity against a variety of tumors.

Methods: We selected OSCC CAL-27 cells as the in vitro model. Cell toxicity, proliferation, apoptosis, and stemness were conducted, respectively. The protein levels of Ki67, PCNA, caspase-3, caspase-9, survivin, SOX2, NANOG, OCT4, STAT3, p-STAT3, ERK1/2, and p-ERK1/2 were evaluated by western blotting. Male BALB/c nude mice xenograft model was used to evaluate the effect of Bupi on tumor growth in vivo.

Results: Compared with the control group, Bupi $(0.2,0.5$, or $1 \mu \mathrm{m})$ significantly decreased the cell viability and the proliferation of CAL-27 cells. Meanwhile, Bupi significantly promoted apoptosis of CAL-27 cells compared with the control group. Additionally, Bupi inhibited the stemness of CAL-27 cells which was evidenced by a sphere formation assay. Bupi decreased the phosphorylation level of STAT3 and ERK1/2 in a dose-dependent manner. The addition of interferon- $\gamma(\mathrm{IFN}-\gamma, 20 \mathrm{ng} / \mathrm{mL})$ in the experiment verified the role of Bupi on STAT3 and ERK1/2 signaling. In vivo, Bupi $(40 \mu \mathrm{mol} / \mathrm{kg})$ obviously suppressed the weight and size of the xenograft tumor, the number of apoptotic cells and Ki67+ decreased. Also, Bupi treatment inhibited the expression of stem-like marker proteins.

Conclusions: Bupi could be used as an anticancer drug against the growth and stemness ability of OSCC. The underlying mechanism may be due to down-regulation of STAT3 and ERK1/2 signaling. This study provides a new insight for the application of Bupi.

Keywords: Bupivacaine (Bupi); malignant tumor; STAT3; ERK; stemness

Submitted Jan 14, 2021. Accepted for publication Apr 29, 2021.

doi: $10.21037 / \mathrm{atm}-21-1414$

View this article at: http://dx.doi.org/10.21037/atm-21-1414

\section{Introduction}

Oral squamous cell carcinoma (OSCC) is the main type of oral cancer, accounting for nearly $90 \%$ of cases (1). According to reports, the incidence of OSCC is increasing yearly. By the end of 2017, it was estimated that the global number of new cases and deaths caused by OSCC were $1,688,780$ and 600,920 , respectively $(2,3)$. The high incidence of OSCC is mainly attributed to two major risk factors: smoking and drinking (4). Also, OSCC is associated with long-term malnutrition, oral viral infection, and poor 
hygiene (5). Despite the tremendous progress made in medical treatment, the five-year survival rate for OSCC has hardly improved in the past few decades (6). Therefore, it is vitally important to identify the intrinsic mechanism of OSCC and seek new therapeutic strategies for its treatment.

In clinical applications, local anesthetics are used for local infiltration, peripheral nerve blockade, and central neuraxial anesthesia in cancer surgery. Local anesthesia is part of the patient's preoperative analgesic regimen. It has been reported that using local anesthetics clinically could decrease recurrence of breast cancer (7-9), thyroid cancer (10), prostate cancer (11), and OSCC (12). Bupivacaine (Bupi) is a widely used long-acting local anesthetic. Strikingly, in the last decade, Bupi has been reported to inhibit the development of various tumors (13-15). Still, the anti-OSCC role of Bupi has not been discussed yet, and our work revolves around the anti-tumor effects of Bupi in oral squamous cell carcinoma.

The treatment of OSCC has been developing for decades. Targeting cell proliferation and stemness are valid cytotoxic strategies on human OSCC cells (16). Signal transducer and activator of transcription 3 (STAT3) is a member of the STAT family and participates in many physiological processes including cell proliferation and differentiation (17). In head and neck squamous cell carcinoma, phosphorylated STAT3 was associated with lymph node metastasis, recurrence, and poor prognosis (18). Extracellular signal-regulated kinase (ERK) is a subfamily of the mitogen-activated protein kinase. ERK was reported to be implicated in OSCC development and progression (19). Thus, activation of ERK1/2 and STAT3 could contribute to the development of OSCC (20). A positive correlation between ERK/STAT3 activation and OSCC was found in several studies $(19,21)$. Our aim in this study was to evaluate the activation levels of STAT3 and ERK1/2 in OSCC cells and investigate the possible correlations of these molecules with the malignant progression of OSCC.

In the present study, relevant research was conducted to explore the role of Bupi on OSCC cells and its underlying mechanism of action.

We present the following article in accordance with the MDAR reporting checklist (available at http://dx.doi. org/10.21037/atm-21-1414).

\section{Methods}

\section{Cell culture}

The human OSCC cell line (CAL-27) was obtained from the American Type Culture Collection (ATCC, Manassas, VA, USA) and suspended in Dulbecco's Modified Eagle Medium (DMEM; Gibco Inc., Billings, MT, USA) supplemented with $10 \%$ fetal bovine serum (FBS; Gibco), $100 \mathrm{U} / \mathrm{mL}$ penicillin $+100 \mathrm{mg} / \mathrm{mL}$ streptomycin (Gibco). The CAL-27 cells were cultured in a $5 \% \mathrm{CO}_{2}$ humidified incubator at $37^{\circ} \mathrm{C}$. Bupi was obtained from ApexBio China (Shanghai, China).

\section{Cell toxicity assay}

The CAL-27 cell toxicity of the Bupi assay was performed by a cell counting kit-8 (CCK-8; Beyotime, Haimen, China), according to the manufacturer's instructions. Bupi (molecular formula: $\mathrm{C}_{18} \mathrm{H}_{28} \mathrm{~N}_{2} \mathrm{O}$, molecular weight: 288.4, purity: $98.00 \%$, Figure $1 A$ ) was purchased from Glpgio Technology (Shanghai, China). The CAL-27 cell cultures were treated with a Bupi concentration gradient ranging from 0 to $100 \mu \mathrm{m}$ for $48 \mathrm{~h}$. The concentration gradient was selected in accordance with previous literature (22).

\section{5-ethynyl-2-deoxyuridine (EdU) staining assay}

The CAL-27 cells in each group were cultured in a 24-well plate and treated with $50 \mu \mathrm{mol} / \mathrm{L} \mathrm{EdU} \mathrm{medium} \mathrm{for}$ $2 \mathrm{~h}$. The EdU incorporation assay was performed by a CellLight ${ }^{\mathrm{TM}}$ EdU imaging kit, according to the manufacturer's instructions (RiboBio, Guangzhou, China). After fixing with $4 \%$ paraformaldehyde, the cells were stained with the EdU reaction solution, then the cell nucleus was stained with Hoechst.

\section{Flow cytometry}

The CAL-27 cell apoptotic rate was detected by an Annexin V-FITC/propidium iodide (PI) apoptosis detection kit (Multisciences, Shanghai, China) according to the manufacturer's instructions. Briefly, after washing twice with ice-cold PBS, CAL-27 cells were re-suspended and stained with $5 \mu \mathrm{L}$ of Annexin V-FIFC and $10 \mu \mathrm{L}$ of PI for 15 min under dark conditions. The samples were analyzed in a fluorescence-activated cell sorting (FACS) Calibur flow cytometer (BD, Franklin Lakes, NJ, USA).

\section{Western blotting analysis}

The CAL-27 cells were collected and treated with radioimmunoprecipitation assay (RIPA) lysis buffer containing 
A

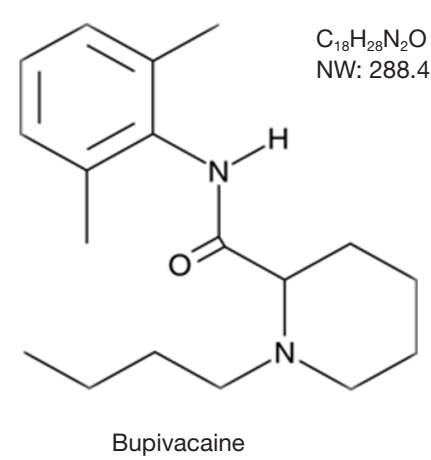

B

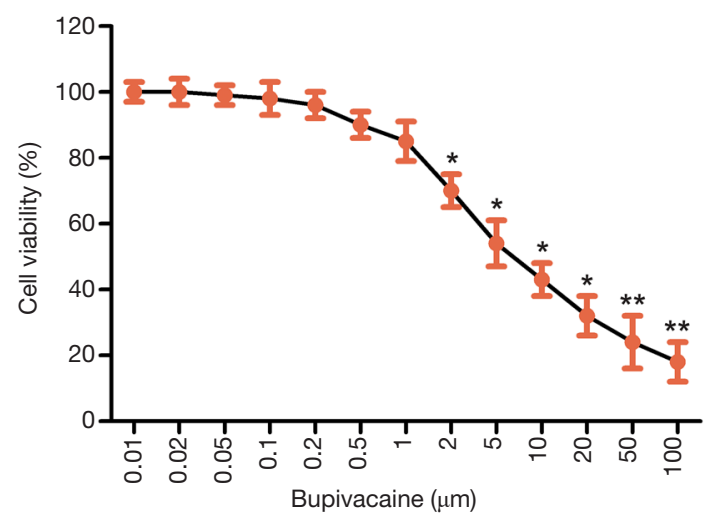

Figure 1 Bupi inhibits the viability of CAL-27 cells. (A) Chemical structure of Bupi. (B) Cell viability of CAL-27 cells is examined using a CCK-8 assay. Cell viability $(\%)=(\mathrm{OD} 490$ of the samples/OD 490 of the control $) \times 100 \% .{ }^{*} \mathrm{P}<0.05,{ }^{* *} \mathrm{P}<0.01$ vs. 0 h. Data are presented as mean \pm SD in triplicate. CCK-8, cell counting kit-8; OD, optical density.

protease inhibitor. Cell samples were quantified by a bicinchoninic acid (BCA) kit (Beyotime). Then, the total proteins were separated by a sodium dodecyl sulfate (SDS)polyacrylamide gel and transferred to polyvinylidene difluoride (PVDF) membranes (Millipore, Billerica, MA, USA). After sealing with 5\% non-fat milk in Tris buffered saline Tween (TBST), the membranes were incubated with specific primary antibodies overnight at $4{ }^{\circ} \mathrm{C}$. The primary antibodies used were as follows: Ki67 (ab16667, Abcam, UK), PCNA (\#2586, cell signaling technology, CST, USA), caspase-3 (ab13847, Abcam), caspase-9 (ab32539, Abcam), survivin (ab76424, Abcam), SOX2 (ab97959, Abcam), NANOG (ab109250, Abcam), OCT4 (ab18976, Abcam), STAT3 (ab119352, Abcam), p-STAT3 (ab76315, Abcam), ERK1/2 (ab17942, Abcam), p-ERK1/2 (ab223500, Abcam). On the next day, the horseradish peroxidase-conjugated secondary antibody anti-mouse IgG (\#7076, CST) or antirabbit IgG (\#7074, CST) were incubated for $2 \mathrm{~h}$ at room temperature. Finally, the targeted strips were visualized using an electrochemiluminescence (ECL) solution. Glyceraldehyde-3-phosphate dehydrogenase (GAPDH) was used as an internal control.

\section{Cell sphere formation assay}

Approximately $1.5 \times 10^{3}$ CAL-27 cells were seeded in six-well plates (Corning, NY, USA) and cultured in DMEM-F12 medium (serum-free) supplemented with $20 \mathrm{ng} / \mathrm{mL}$ EGF, $10 \mathrm{ng} / \mathrm{mL}$ bFGF, N-2, and 1\% B27 (Sigma-Aldrich, St.
Louis, USA). Ten days later, the plate was subjected to the microscope image. The number and diameter (diameter $>10 \mu \mathrm{m}$ ) of spheres were captured from five images using an inverted microscope (Zeiss, Oberkochen, Germany).

\section{Analysis of tumorigenicity in vivo}

A total of $30 \mathrm{BALB} / \mathrm{c}$ nude mice (4-week-old) were used to established xenograft nude model. About $1 \times 10^{7} \mathrm{CAL}-27$ cells were injected subcutaneously, the mice were treated with bupivacaine $(40 \mu \mathrm{mol} / \mathrm{kg})$ or equal volume saline. After 7 days of injection, we measured tumor growth every 3 days. 30 days later, the mice were killed by cervical dislocation, and tumors were harvested to assess tumor weight or size, followed by immunohistochemical and western blotting analysis. Animal care and method procedure were authorized by the Animal Ethics Committee of The Affiliated Hospital of Stomatology of Southwest Medical University (No. 20180722001), and were carried out in accordance with the National Institutes of Health guide for the care and use of Laboratory animals (National Academy of Sciences Press, 2011).

\section{Statistical analysis}

Two-group or multiple comparisons were analyzed by oneway ANOVA followed by Tukey's post-hoc tests. Data are represented as the mean \pm SD. Statistical significance was indicated if $\mathrm{P}<0.05$. The statistical analysis was performed 
using SPSS 25.0 (IBM, Armonk, NY, USA).

\section{Results}

Bupi inbibited the viability of CAL-27 cells

A CCK-8 assay was employed to detect the cytotoxicity of CAL-27 cells treated with Bupi at a concentration gradient from 0 to $100 \mu \mathrm{m}$ for $24 \mathrm{~h}$. The result indicated that Bupi had an inhibitory action on CAL-27 cells in a dosedependent manner (Figure 1B). However, at concentrations higher than $2 \mu \mathrm{m}$, Bupi exhibited obvious cytotoxicity to CAL-27 cells. Therefore, we chose 3 concentrations (0.2, 0.5 , or $1 \mu \mathrm{m}$ ) for subsequent experiments.

\section{Bupi inbibited the proliferation capability of CAL-27 cells}

To discover Bupi's role on proliferation, EdU and clonal formation assays were performed. As shown in Figure 2A, the number of EdU positive cells (shown as red) was significantly decreased by Bupi treatment in a dosedependent manner $(\mathrm{P}<0.05)$, compared with the control group. The cell colony formation rate was significantly reduced compared with the control group (Figure 2B). In addition, western blotting results showed that Bupi downregulated the Ki67 and PCNA maker protein expressions in a dose-dependent manner (Figure $2 C$, $\mathrm{P}<0.05)$.

\section{Bupi stimulated the apoptosis of CAL-27 cells}

To elucidate the role of Bupi on apoptosis, flow cytometry was performed. As seen in Figure $3 A, B$, the cell apoptosis rate was significantly increased by Bupi treatment in a dosedependent manner $(\mathrm{P}<0.05)$. Furthermore, the expression of caspase-3, caspase-9, and survivin proteins were detected. Results showed that Bupi downregulated the survivin protein expression but upregulated the caspase- 3 and caspase-9 protein expressions in a dose-dependent manner (Figure $3 C, D, \mathrm{P}<0.05$ ).

\section{Bupi suppressed the stemness of CAL-27 cells}

To clarify the role of Bupi on the stemness of CAL-27 cells, a cell sphere formation assay was performed as previously described. As shown in Figure 4A, Bupi suppressed the formation of spheres by reducing the diameters of the spheres (Figure 4B) and the sphere numbers (Figure 4C) in a dose-dependent manner. Also, we detected the expression of stemness marker proteins, including SOX2, NANOG, and OCT4. Results showed that SOX2, NANOG, and OCT4 protein expressions were all down-regulated by Bupi in a dose-dependent manner. (Figure 4D,E, $\mathrm{P}<0.05$ ). These data indicated that Bupi clearly reduced the stemness of CAL-27 cells.

\section{Bupi inhibited the phosphorylation levels of ERK1/2 and STAT3}

To elucidate the underlying anticancer mechanism of Bupi, the expression levels of ERK1/2 and STAT3 as well as their phosphorylation forms were detected. As shown in Figure 5A, western blotting analysis showed that Bupi downregulated the phosphorylation level of STAT3 in a dose-dependent manner $(\mathrm{P}<0.05)$. In addition, the phosphorylation level of ERK1/2 (p-ERK1/2) was decreased by Bupi in a dose-dependent manner (Figure $5 B$, $\mathrm{P}<0.05)$. In particular, Bupi inhibited the upregulation of p-ERK1/2 and p-STAT3 induced by interferon- $\gamma$ (IFN- $\gamma$, $20 \mathrm{ng} / \mathrm{mL}$ ) (Figure $5 C, D, \mathrm{P}<0.05$ ). These data indicated that Bupi significantly inhibited the phosphorylation of ERK1/2 and STAT3 in CAL-27 cells.

\section{Bupi decreased carcinogenicity and stemness by inbibiting ERK1/2 and STAT3 activation}

IFN- $\gamma$ was added to the experiments to clarify whether Bupi regulated proliferation, apoptosis, and stemness by inhibiting STAT3 and ERK1/2 signaling. As shown in Figure $6 A, B, 1 \mu \mathrm{m}$ of Bupi significantly reversed the role of IFN- $\gamma(20 \mathrm{ng} / \mathrm{mL})$ in promoting cell proliferation $(\mathrm{P}<0.05)$. Moreover, $1 \mu \mathrm{m}$ of Bupi clearly stimulated the apoptosis inhibited by IFN- $\gamma$ (Figure $6 A, C, \mathrm{P}<0.05$ ). Importantly, $1 \mu \mathrm{m}$ of Bupi downregulated the stemness marker proteins SOX2, NANOG, and OCT4 induced by IFN- $\gamma$ (Figure $6 D, E, \mathrm{P}<0.05$ ). These data indicated that Bupi regulated proliferation, apoptosis, and stemness via STAT3 and ERK1/2 signaling.

\section{Bupi inbibited tumor enlargement in tumor-bearing mice}

CAL-27 cells were subcutaneously injected to form xenograft tumors in vivo. The results showed that tumor weight and volume treated with $40 \mu \mathrm{mol} / \mathrm{kg}$ Bupi were obviously lower compared with the control group 
A

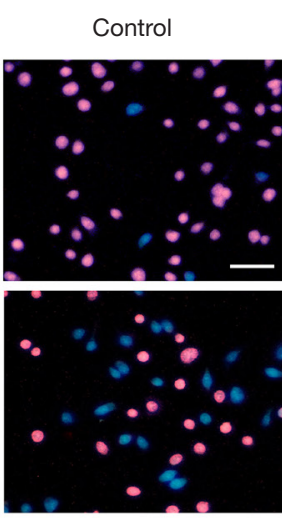

Bupi $0.5 \mu \mathrm{m}$

B

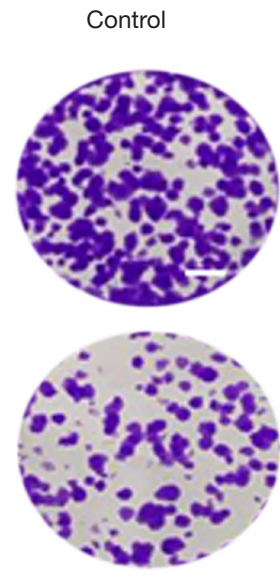

Bupi $0.5 \mu \mathrm{m}$
Bupi $0.2 \mu \mathrm{m}$

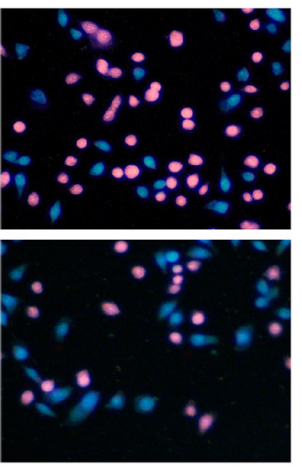

Bupi $1 \mu \mathrm{m}$
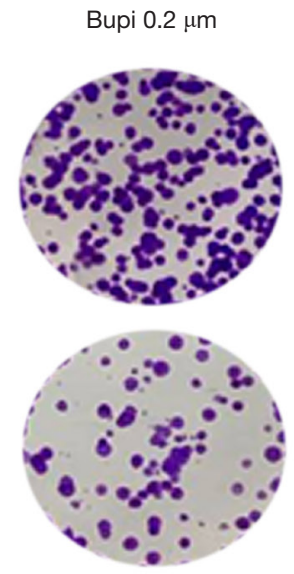

Bupi $1 \mu \mathrm{m}$
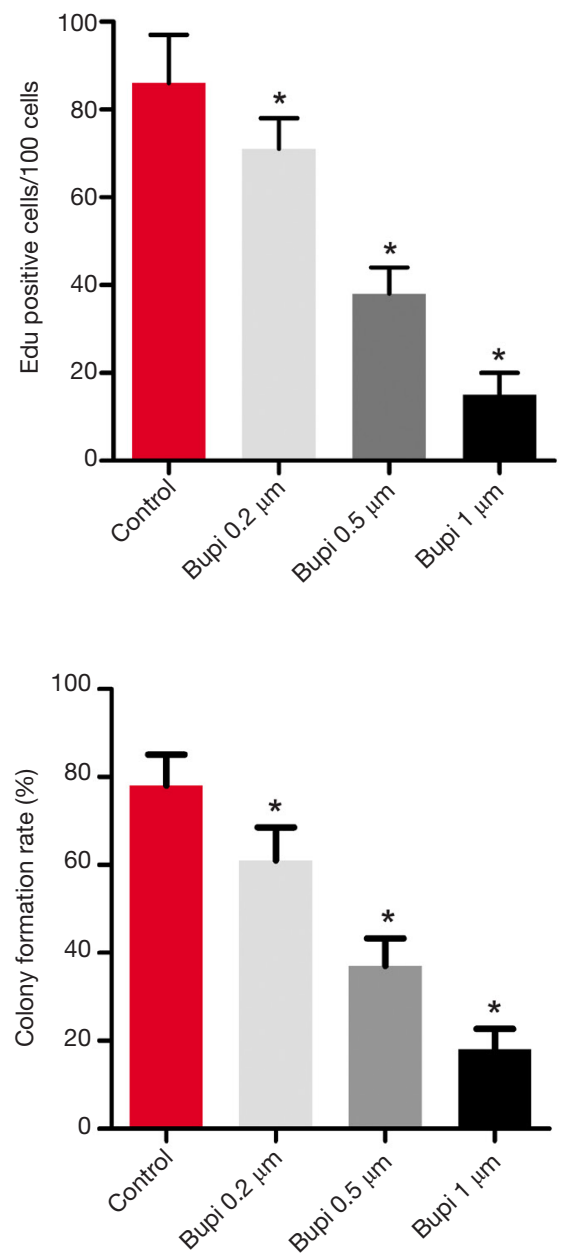

C

Control

$\square$ Bupi $0.2 \mu \mathrm{m}$

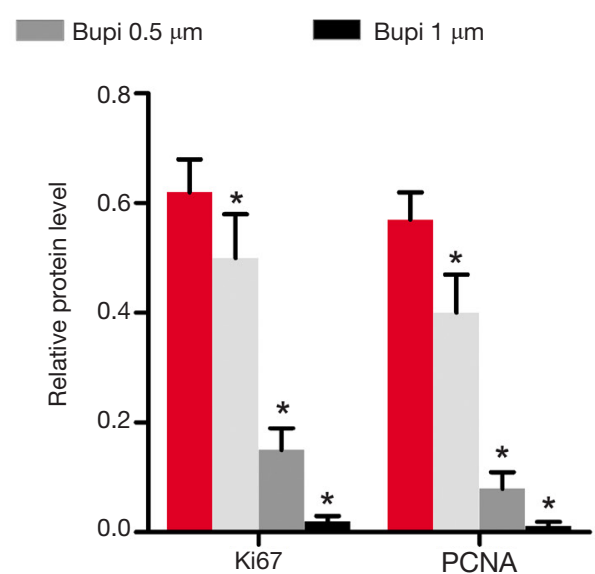

Figure 2 Bupi inhibits cell proliferation of CAL-27 cells. (A) EdU staining and (B) clone formation assay showing the effect of Bupi on the proliferation ability of CAL-27 cells. The EdU positive cells are photographed and counted. The scale bar $=50 \mu \mathrm{m}$. (C) The relative expression of Ki67 and PCNA proteins are detected using western blotting. ${ }^{*} \mathrm{P}<0.05 v$ s. control. Data are presented as mean $\pm \mathrm{SD}$ in triplicate. EdU, 5-ethynyl-2-deoxyuridine; PCNA, proliferating cell nuclear antigen. 
A
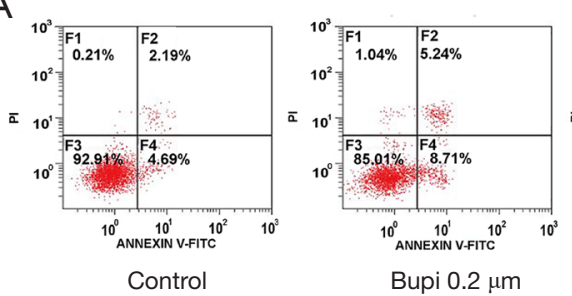

C

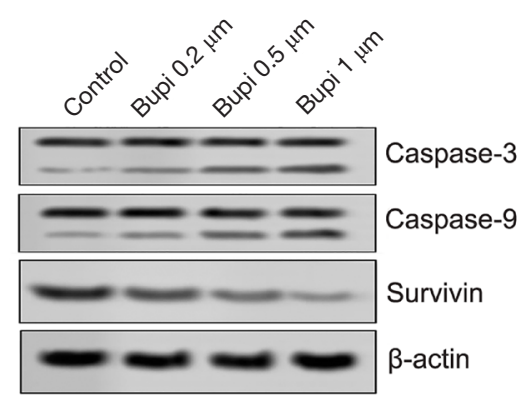

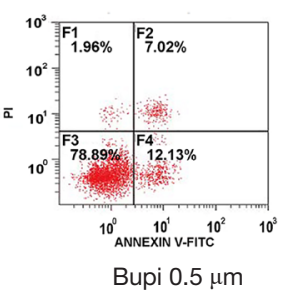

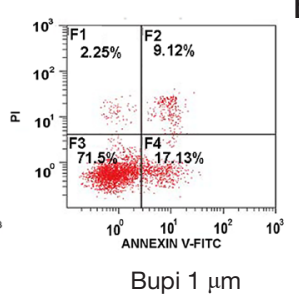

D

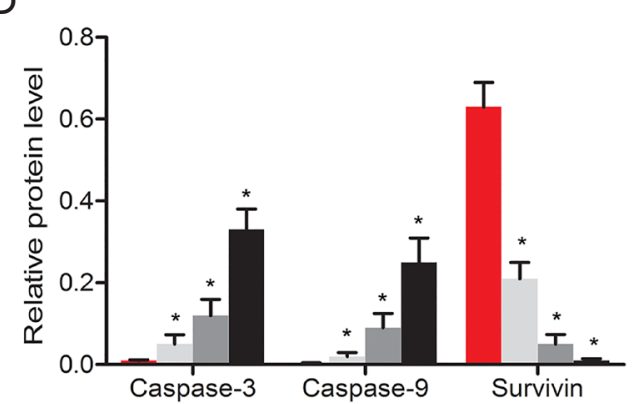

B
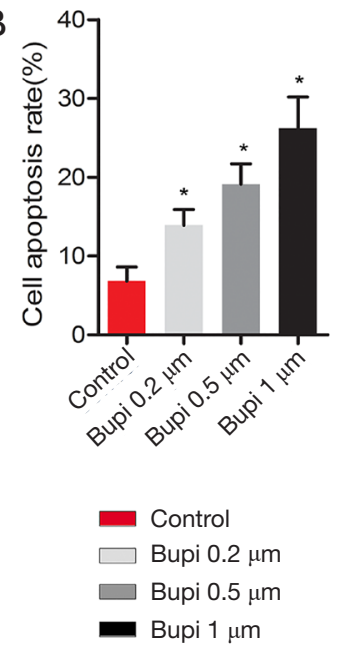

Bupi $1 \mu \mathrm{m}$

Figure 3 Bupi induces the apoptosis of CAL-27 cells. (A,B) Flow cytometry assay showing the effect of Bupi on cell apoptosis in CAL27 cells. The cell apoptosis rate is quantified by counting the Q2 + Q4 quadrant. (C,D) The relative expression of cleaved caspase-3/9 and survivin proteins is examined by western blotting. ${ }^{*} \mathrm{P}<0.05 v$ s. control. Data are presented as mean $\pm \mathrm{SD}$ in triplicate.

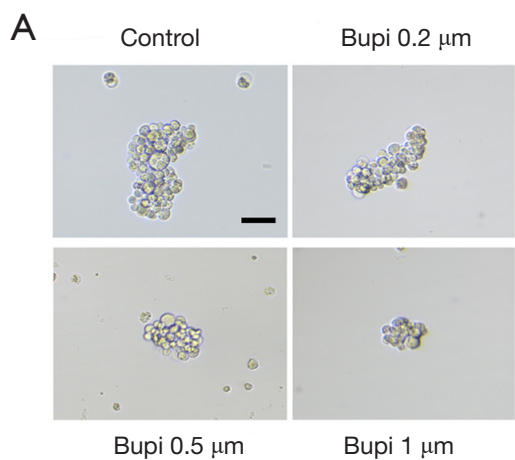

D

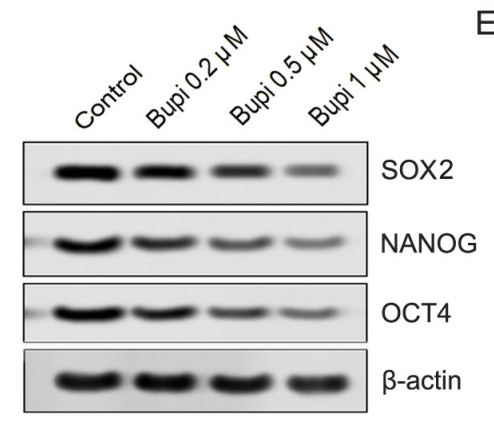

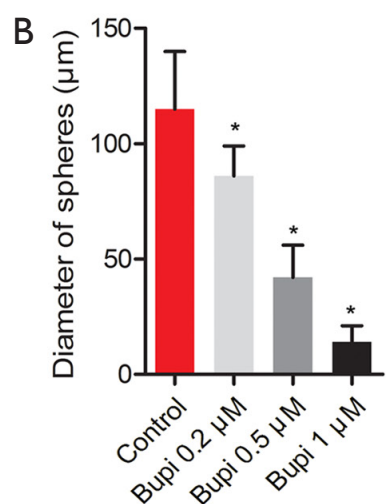

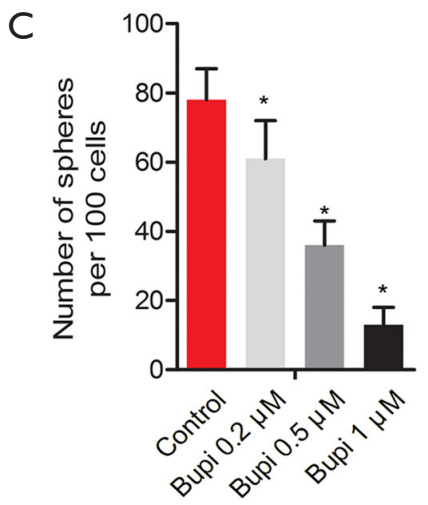

$E$

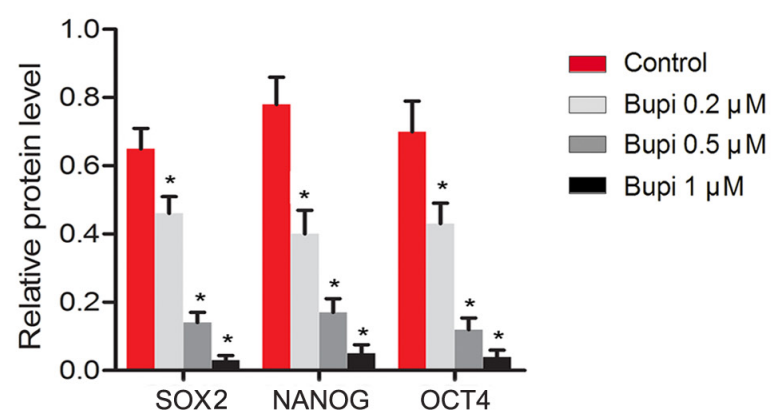

Figure 4 Bupi inhibits the cell stemness of CAL-27 cells. (A) Cell stemness of CAL-27 cells is assessed by sphere formation assay. The scale bar $=50 \mu \mathrm{m}$. (B) The diameters of spheres in each group are measured. (C) The number of spheres in each group are counted. (D,E) The expression of stemness-related proteins is determined by western blotting. ${ }^{*} \mathrm{P}<0.05$ vs. control. Data are presented as mean $\pm \mathrm{SD}$ in triplicate. SOX2, sex determining region of Ychromosome related high mobility group box 2; OCT4, recombinant octamer binding transcription factor 4. 
A

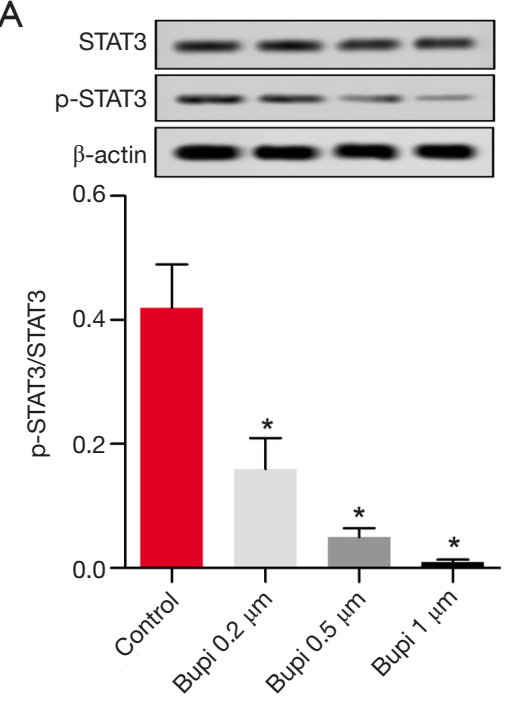

C

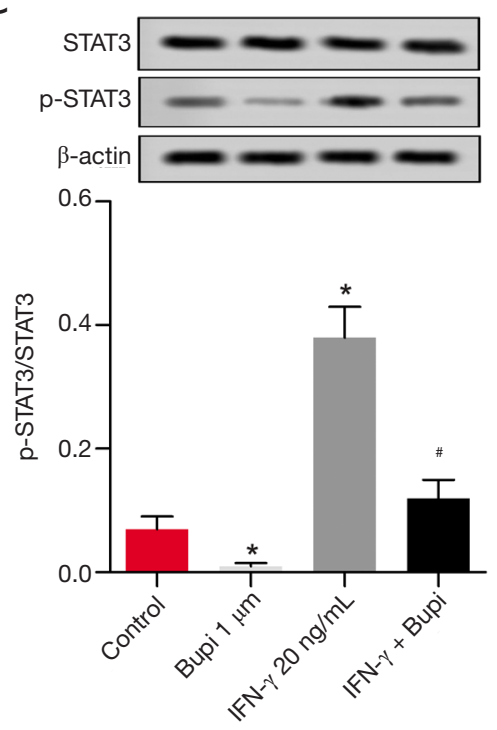

B
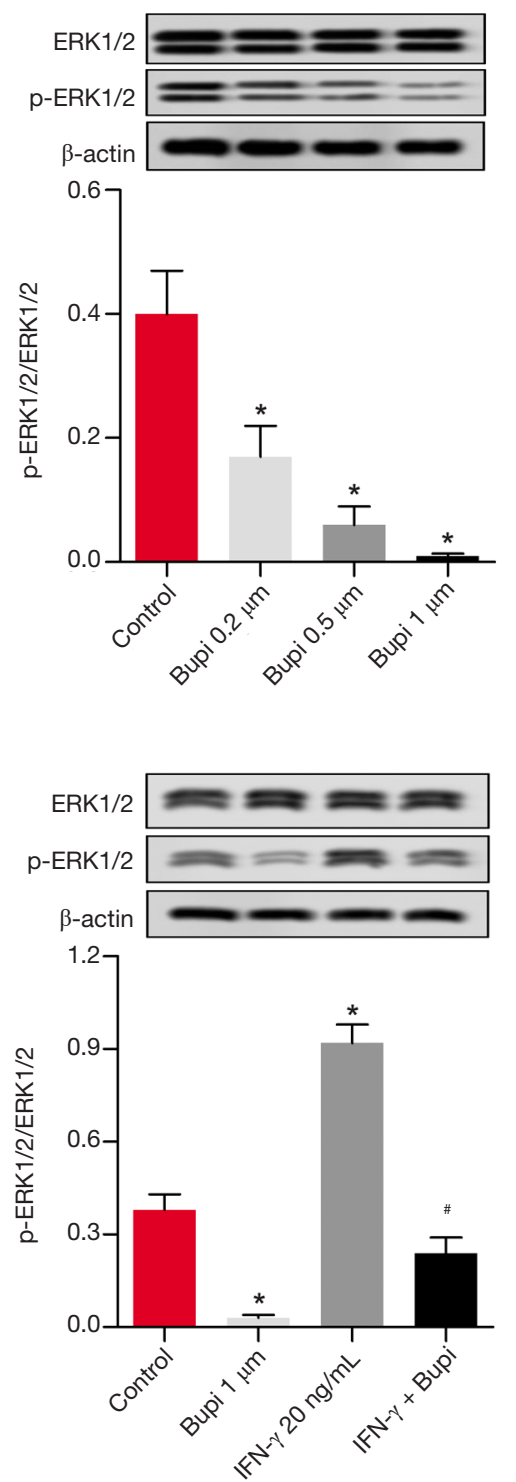

Figure 5 Bupi suppresses the phosphorylation of ERK1/2 and STAT3. (A) The protein expression of STAT3 and p-STAT3 is examined using western blotting. (B) The protein expression of ERK1/2 and p- ERK1/2 in CAL-27 cells is examined using western blotting. Cells are grouped into control, Bupi $1 \mu \mathrm{m}, \mathrm{IFN}-\gamma 20 \mathrm{ng} / \mathrm{mL}$, and Bupi + IFN- $\gamma$. (C) The relative expressions of STAT3 and p-STAT3 are detected by western blot. (D) The relative expression of ERK1/2 and p-ERK1/2 in CAL-27 cells is detected by western blot. ${ }^{*} \mathrm{P}<0.05$ vs. control, ${ }^{\#} \mathrm{P}<0.05$ $v s$. IFN- $\gamma$. Data are presented as mean \pm SD in triplicate. STAT3, signal transducer and activator of transcription 3; ERK, extracellular signal-regulated kinase; IFN- $\gamma$, interferon- $\gamma$.

(Figure $7 A, B$ ). The apoptotic cells were increased, and the number of $\mathrm{Ki}^{\circ} 7^{+}$expression was decreased (Figure $7 C, D, E$ ). Neferine treatment also down-regulated the expression of SOX2, NANOG, and OCT4 proteins (Figure 7F).

\section{Discussion}

Due to the high incidence of recurrence and the lack of effective early treatment strategies, the treatment of OSCC remains a huge challenge (23). The pathology mechanism 
A

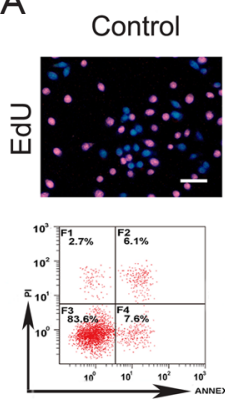

Control
Bupi $1 \mu \mathrm{M}$
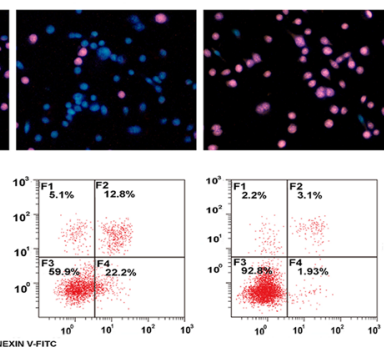

Bupi $1 \mu \mathrm{M}$

D

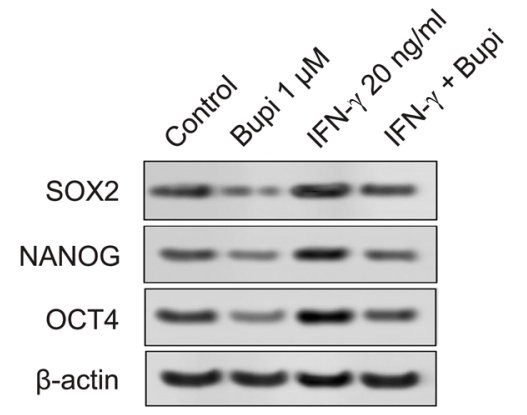

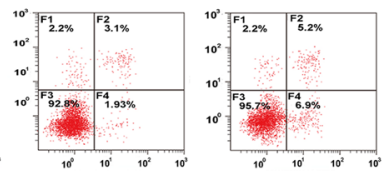

IFN- $\gamma 20 \mathrm{ng} / \mathrm{mL} \quad$ IFN- $\gamma+$ Bupi

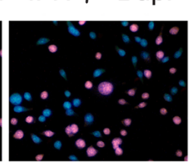

B

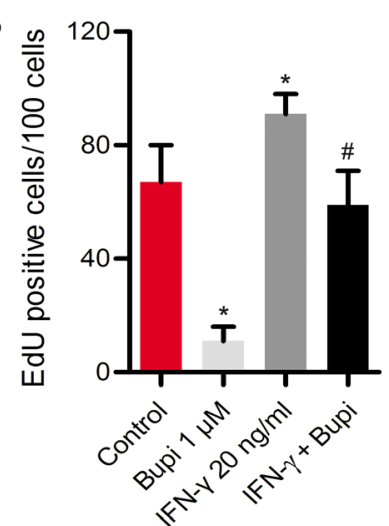

$E$

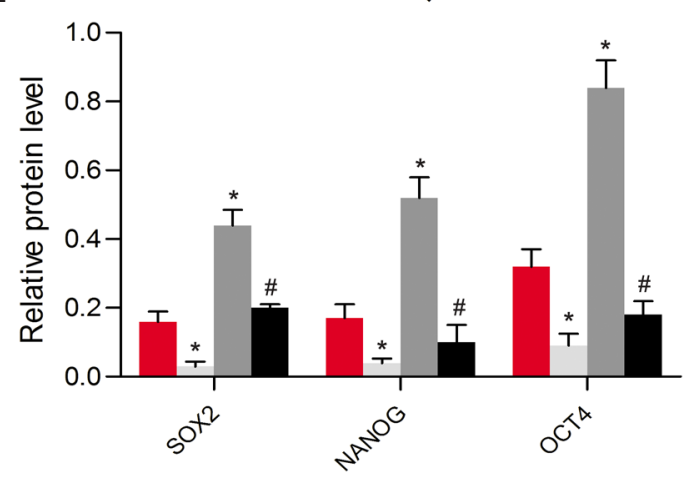

C

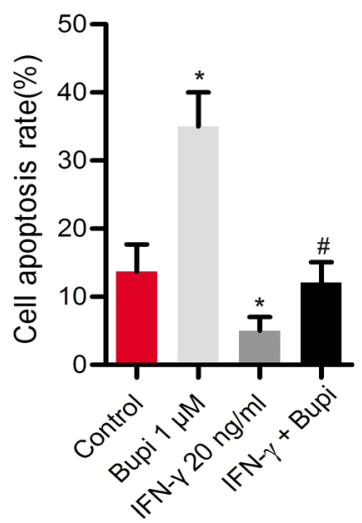

Control

Bupi $1 \mu \mathrm{M}$

IFN- $\gamma 20 \mathrm{ng} / \mathrm{mL}$

IFN- $\gamma+$ Bupi

Figure 6 Bupi decreases the carcinogenicity and stemness of OSCC cells by inhibiting ERK1/2 and STAT3 activation. (A) EdU staining assay showing the effect of Bupi on the proliferation ability of CAL-27 cells. The scale bar $=50 \mu$ m; flow cytometry assay showing the effect of Bupi on the apoptosis of CAL-27 cells. (B) The EdU positive cells are photographed and counted. (C) The cell apoptosis rate is quantified by counting the Q2+Q4 quadrant. (D) The expression of stemness-related proteins are determined by western blotting. (E) The representative bar graph showing results of the relative protein expression. ${ }^{*} \mathrm{P}<0.05$ vs. control, ${ }^{\#} \mathrm{P}<0.05$ vs. IFN- $\gamma$. Data are presented as mean \pm SD in triplicate. IFN- $\gamma$, interferon- $\gamma$; SOX2, sex determining region of Ychromosome related high mobility group box 2; OCT4, recombinant octamer binding transcription factor 4.

of OSCC is related to the unlimited proliferation and stemness formation of oral squamous cells $(24,25)$. In the current work, CAL-27 cells were selected as the object of an in vitro study to explore the role of Bupi on OSCC.

Bupi has been shown to limit the growth and migration of several cancer cells. For example, Bupi was reported to inhibit cell viability and proliferation and promote apoptosis in thyroid cancer by inhibiting ERK1/2 signaling (10). Li et al. indicated that Bupi impeded the cell viability and migration of breast cancer (7). Moreover, A spectrum of studies reported that Bupi induced cell apoptosis in breast cancer cells (9), ovarian cancer cells (26), and OSCC (12). Consistent with previous studies, our primary findings indicated that Bupi can inhibit the proliferative capacity and induce apoptosis in OSCC CAL-27 cells. Notably, our study also revealed that Bupi can impede the stemness of cancer cells. It has been suggested that the tumorigenic abilities and resistance to conventional chemotherapy and radiation of OSCC cells are due to their stemness (27). Therefore, inhibiting OSCC cell stemness is a promising strategy to delay the development of OSCC. In this study, Bupi suppressed the formation of spheres, and the stemness-related proteins SOX2, NANOG, and OCT4 were significantly downregulated, which suggests that Bupi can inhibit the stemness of OSCC CAL-27 cells.

STAT signals have been found to be associated with tumorigenesis (28). STAT3 sustained activation contributes to malignant phenotype $(18,29)$. It has also been shown that STAT3 promotes initiation and malignant progression of OSCC (30,31). Previous reports support the activation of MAPK family-specific members negatively regulates signals in STAT3 various cell types $(32,33)$. Studies have 

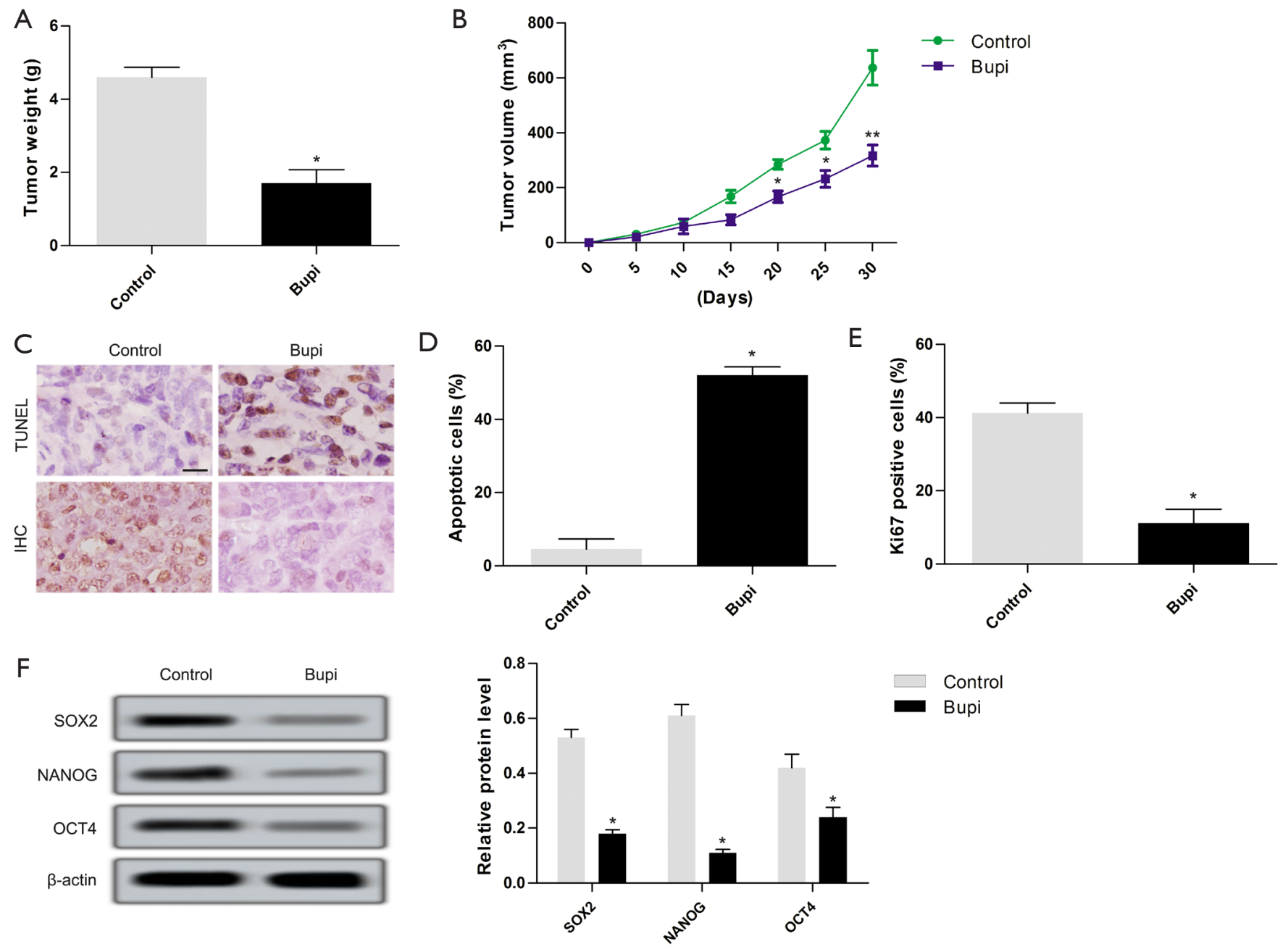

Figure 7 Bupi inhibits tumorigenesis of xenograft tumors in vivo. OSCC tumor-bearing model was established with $40 \mu \mathrm{mol} / \mathrm{kg}$ Bupi. (A) Tumor weight. (B) Tumor volume. (C,D,E) The number of apoptotic cells was detected by TUNEL staining; Ki67+ expression detected by IHC staining; representative images were magnified at 400x. (F) The relative expressions of SOX2, NANOG, and OCT4 were detected by western blot. ${ }^{*} \mathrm{P}<0.05$ vs. control. Data are presented as mean $\pm \mathrm{SD}$ in triplicate. TUNEL, terminal deoxynucleotidyl transferase-mediated dUTP nick-end labeling; IHC, immunohistochemical; SOX2, sex determining region of Ychromosome related high mobility group box 2; OCT4, recombinant octamer binding transcription factor 4.

shown that ERK can phosphorylate serine 727 (Ser727) in vivo and in vitro while inhibiting STAT3 phosphorylation of tyrosine (34). Consistent with previous reports, we explored the effect of Bupi on the activity of ERK1/2 and STAT3. In this study, Bupi treatment significantly inhibited the generation of pERK1/2 and pSTAT3, which suggested that Bupi could inhibit ERK and STAT3 signaling pathways.

IFN- $\gamma$ is a cytokine secreted by $\mathrm{CD} 8^{+}$cytotoxic T lymphocytes, which are natural killer cells and macrophages. IFN- $\gamma$ regulates the differentiation to inhibit the growth and survival of cancer cells (35). However, increasing evidence has shown that IFN- $\gamma$ is a cytokine with both anticancer and carcinogenic functions $(36,37)$. IFN- $\gamma$ is generally regarded as an inflammatory cytokine and serves as an inducer of the activation of ERK and STAT3 signaling (38). Hence, in this paper, we used IFN- $\gamma$ as an inducer of ERK1/2 and STAT3 phosphorylation. Our findings indicated that IFN- $\gamma$ could enhance the proliferation and stemness, as well as decrease the apoptosis, of OSCC CAL-27 cells. Of note, Bupi counteracted the effect of IFN- $\gamma$ on cell proliferation, apoptosis, and stemness, which suggested that Bupi regulated the cell 
proliferation, apoptosis, and stemness of OSCC CAL27 cells by suppressing the activation of ERK and STAT3 signaling.

In summary, our findings showed that Bupi can suppress the viability, cell proliferation, and stemness of OSCC CAL-27 cells. The antitumor mechanism may involve the inhibition of ERK and STAT3 signaling pathway activation. This study provides a new insight for OSCC treatment and enriches our understanding of the pharmacological actions of Bupi.

\section{Acknowledgments}

Funding: National Key Laboratory for Virtual Reality Technology and Systems (VR):(VRLAB2020D05) and the Sichuan Health and Family Planning Commission (18 PJ097).

\section{Footnote}

Reporting Checklist: The authors have completed the MDAR reporting checklist. Available at http://dx.doi.org/10.21037/ atm-21-1414

Data Sharing Statement: Available at http://dx.doi. org/10.21037/atm-21-1414

Conflicts of Interest: All authors have completed the ICMJE uniform disclosure form (available at http://dx.doi. org/10.21037/atm-21-1414). The authors have no conflicts of interest to declare.

Ethical Statement: The authors are accountable for all aspects of the work in ensuring that questions related to the accuracy or integrity of any part of the work are appropriately investigated and resolved. Animal care and method procedure were authorized by the Animal Ethics Committee of The Affiliated Hospital of Stomatology of Southwest Medical University (No. 20180722001), and were carried out in accordance with the National Institutes of Health guide for the care and use of Laboratory animals (National Academy of Sciences Press, 2011).

Open Access Statement: This is an Open Access article distributed in accordance with the Creative Commons Attribution-NonCommercial-NoDerivs 4.0 International License (CC BY-NC-ND 4.0), which permits the noncommercial replication and distribution of the article with the strict proviso that no changes or edits are made and the original work is properly cited (including links to both the formal publication through the relevant DOI and the license). See: https://creativecommons.org/licenses/by-nc-nd/4.0/.

\section{References}

1. Liang X, Deng M, Zhang C, et al. Combined class I histone deacetylase and mTORC1/C2 inhibition suppresses the initiation and recurrence of oral squamous cell carcinomas by repressing SOX2. Cancer Lett 2019;454:108-19.

2. He B, Lin XZ, Tian FQ, et al. MiR-133a-3p inhibits oral squamous cell carcinoma (OSCC) proliferation and invasion by suppressing COL1A1. J Cell Biochem 2018;119;338-46.

3. Siegel RL, Miller KD, Jemal A. Cancer statistics, 2018. CA Cancer J Clin 2018;68:7-30.

4. Tanaka F, Yamamoto K, Suzuki S, et al. Strong interaction between the effects of alcohol consumption and smoking on oesophageal squamous cell carcinoma among individuals with ADH1B and/or ALDH2 risk alleles. Gut 2010;59:1457-64.

5. van Zyl A, Bunn BK. Clinical features of oral cancer. SADJ 2012;67:566-9.

6. Marsh D, Suchak K, Moutasim KA, et al. Stromal features are predictive of disease mortality in oral cancer patients. J Pathol 2011;223:470-81.

7. Li R, Xiao CY, Liu HR, et al. Effects of local anesthetics on breast cancer cell viability and migration. BMC cancer 2018;18:666.

8. Ni J, Xie T, Xiao MF, et al. Amide-linked local anesthetics preferentially target leukemia stem cell through inhibition of Wnt/ $\beta$-catenin. Biochem Biophys Res Commun 2018;503:956-62.

9. Chang YC, Liu CL, Chen MJ, et al. Local anesthetics induce apoptosis in human breast tumor cells. Anesth Analg 2014;118:116-24.

10. Chang YC, Hsu YC, Liu CL, et al. Local anesthetics induce apoptosis in human thyroid cancer cells through the mitogen-activated protein kinase pathway. PloS One 2014;9:e89563.

11. Korohoda W, Oczkowicz J, Rolski K, et al. Efficacy of local anesthetics in detachment of normal 3T3mouse fibroblasts and prostate cancer AT-2 cells from substrata, in maintenance of viable cells in a non-adherent state, and in preservation of cell surface markers detected with Flow Sight image cytometry. Folia Biol (Krakow) 
2015;63:249-55.

12. Kobayashi K, Ohno S, Uchida S, et al. Cytotoxicity and type of cell death induced by local anesthetics in human oral normal and tumor cells. Anticancer Res 2012;32:2925-33.

13. Kowalski G, Leppert W, Adamski M, et al. Rectal enema of bupivacaine in cancer patients with tenesmus pain - case series. J Pain Res 2019;12:1847-54.

14. Župčić M, Graf Župčić S, Duzel V, et al. A combination of levobupivacaine and lidocaine for paravertebral block in breast cancer patients undergoing quadrantectomy causes greater hemodynamic oscillations than levobupivacaine alone. Croat Med J 2017;58:270-80.

15. Li T, Chen L, Zhao H, et al. Both Bupivacaine and Levobupivacaine inhibit colon cancer cell growth but not melanoma cells in vitro. J Anesth 2019;33:17-25.

16. Wang LH, Xu M, Fu LQ, et al. The antihelminthic niclosamide inhibits cancer stemness, extracellular matrix remodeling, and metastasis through dysregulation of the nuclear $\beta$-catenin/c-Myc axis in OSCC. Sci Rep 2018;8:12776.

17. Wang Y, Guo W, Li Z, et al. Role of the EZH2/miR200 axis in STAT3-mediated OSCC invasion. Int J Oncol 2018;52:1149-64.

18. Zhao Y, Zhang J, Xia H, et al. Stat3 is involved in the motility, metastasis and prognosis in lingual squamous cell carcinoma. Cell Biochem Funct 2012;30:340-6.

19. Gkouveris I, Nikitakis N, Avgoustidis D, et al. ERK1/2, JNK and STAT3 activation and correlation with tumor differentiation in oral SCC. Histol Histopathol 2017;32:1065-76.

20. Huang WC, Jang TH, Tung SL, et al. A novel miR-365$3 \mathrm{p} / \mathrm{EHF} /$ keratin 16 axis promotes oral squamous cell carcinoma metastasis, cancer stemness and drug resistance via enhancing $\beta 5$-integrin/c-met signaling pathway. J Exp Clin Cancer Res 2019;38:89.

21. Tashiro K, Oikawa M, Miki Y, et al. Immunohistochemical assessment of growth factor signaling molecules: MAPK, Akt, and STAT3 pathways in oral epithelial precursor lesions and squamous cell carcinoma. Odontology 2020;108:91-101.

22. Dan J, Gong X, Li D, et al. Inhibition of gastric cancer by local anesthetic bupivacaine through multiple mechanisms independent of sodium channel blockade. Biomed Pharmacother 2018;103:823-8.

23. Li X, Cheng T, He Y, et al. High glucose regulates ERp29 in hepatocellular carcinoma by LncRNA MEG3-miRNA 483-3p pathway. Life Sci 2019;232:116602.
24. Liu S, Liu L, Hu W, et al. Long noncoding RNA TUG1 regulates the development of oral squamous cell carcinoma through sponging miR-524-5p to mediate DLX1 expression as a competitive endogenous RNA. J Cell Physiol 2019;234:20206-16.

25. Liu X, Fu Y, Huang JD, et al. ADAR1 promotes the epithelial-to-mesenchymal transition and stem-like cell phenotype of oral cancer by facilitating oncogenic microRNA maturation. J Exp Clin Cancer Res 2019;38:315.

26. Xuan W, Zhao HL, Hankin J, et al. Local anesthetic bupivacaine induced ovarian and prostate cancer apoptotic cell death and underlying mechanisms in vitro. Sci Rep 2016;6:26277.

27. Hung KF, Liao PC, Chen CK, et al. ASB6 promotes the stemness properties and sustains metastatic potential of oral squamous cell carcinoma cells by attenuating ER stress. Int J Biol Sci 2019;15:1080-90.

28. Yu H, Kortylewski M, Pardoll D. Crosstalk between cancer and immune cells: role of STAT3 in the tumour microenvironment. Nat Rev Immunol 2007;7:41-51.

29. Haura EB, Turkson J, Jove R. Mechanisms of disease: insights into the emerging role of signal transducers and activators of transcription in cancer. Nat Clin Pract Oncol 2005;2:315-24.

30. Li X, Ding R, Han Z, et al. Targeting of cell cycle and let7a/STAT3 pathway by niclosamide inhibits proliferation, migration and invasion in oral squamous cell carcinoma cells. Biomed Pharmacother 2017;96:434-42.

31. Pouysségur J, Volmat V, Lenormand P. Fidelity and spatio-temporal control in MAP kinase (ERKs) signalling. Biochem Pharmacol 2002;64:755-63.

32. Quadros MR, Peruzzi F, Kari C, et al. Complex regulation of signal transducers and activators of transcription 3 activation in normal and malignant keratinocytes. Cancer Res 2004;64:3934-9.

33. Tkach M, Rosemblit C, Rivas MA, et al. p42/p44 MAPKmediated Stat3Ser727 phosphorylation is required for progestin-induced full activation of Stat 3 and breast cancer growth. Endocr Relat Cancer 2013;20:197-212.

34. Chung J, Uchida E, Grammer TC, et al. STAT3 serine phosphorylation by ERK-dependent and -independent pathways negatively modulates its tyrosine phosphorylation. Mol Cell Biol 1997;17:6508-16.

35. Macha MA, Matta A, Kaur J, et al. Prognostic significance of nuclear pSTAT3 in oral cancer. Head Neck 2011;33:482-9.

36. Zaidi MR, Merlino G. The two faces of interferon- $\gamma$ in 
cancer. Clin Cancer Res 2011;17:6118-24.

37. Mandai M, Hamanishi J, Abiko K, et al. Dual faces of IFN $\gamma$ in cancer progression: A role of PD-L1 induction in the determination of pro- and antitumor immunity. Clin Cancer Res 2016;22:2329-34.

38. Javed Z, Muhammad Farooq H, Ullah M, et al. Wnt

Cite this article as: Wang Z, Zhang J, Wen Y, Wang P, Fan L. Bupivacaine inhibits the malignant biological behavior of oral squamous cell carcinoma cells by inhibiting the activation of ERK1/2 and STAT3. Ann Transl Med 2021;9(10):839. doi: $10.21037 /$ atm-21-1414 signaling: A potential therapeutic target in head and neck squamous cell carcinoma. Asian Pac J Cancer Prev 2019;20:995-1003.

(English Language Editor: D. Fitzgerald) 